Editorial Article

Open Access

\title{
Dengue Fever: An Emerging and Re-emerging Viral disease of Major Public Health Importance
}

\author{
Mahendra Pal ${ }^{\star}$ \\ Founder of Narayan Consultancy on Veterinary Public Health and Microbiology, 4 Aangan, Jagnath Ganesh Dairy Road, Anand-388001, India
}

Article Info

\author{
*Corresponding author: \\ Mahendra Pal \\ Founder of Narayan Consultancy \\ Veterinary Public Health and Microbiology \\ 4 Aangan, Jagnath Ganesh Dairy Road \\ Anand-388001, India \\ E-mail: palmahendra2@gmail.com
}

Received: July 16, 2018

Accepted: July 17, 2018

Published: July 23, 2018

Citation: Pal M. Dengue Fever: An Emerging and Re-emerging Viral disease of Major Public Health Importance. Madridge J Immunol. 2018; 2(1): 51-52

doi: $10.18689 / \mathrm{mjim}-1000112$

\section{Copyright: ( 2018 The Author(s). This work is licensed under a Creative Commons Attribution 4.0 International License, which permits unrestricted use, distribution, and reproduction in any medium, provided the original work is properly cited.}

Published by Madridge Publishers

\section{Introduction}

Emerging and re-emerging infectious diseases are important causes of morbidity and mortality both in developing as well as developed nations. In recent years, dengue fever (break bone fever, dandy fever, dengue haemorragic fever, dengue shock syndrome) has emerged and re-emerged as the most common vector borne disease of major public health concern worldwide. The word dengue originates from denga or dyenga in Africa meaning fever with haemorrhage.

Globally, dengue fever is responsible for 100 million new cases, 500,000 hospitalizations, and 25,000 deaths annually. This vector borne viral disease is more common in the tropical and subtropical regions, and is reported from 100 countries of the world including Bolivia, Brazil, Cambodia, China, Columbia, Cuba, Costa Rica, Ecuador, Hawaii, Honduras, India, Indonesia, Laos, Malaysia, Myanmar, New Guinea, Peru, Philippines, Paraguay, Puerto Rico, Singapore, Sri Lanka, Thailand, Venezuela, and Vietnam. In recent years, epidemics of dengue fever are recorded worldwide. Dengue fever is an emerging viral disease among the US travelers returning from endemic regions. It is reported that around $40 \%$ of the world population living in endemic areas are at risk of dengue virus. There were 80,736 cases of dengue fever with 215 deaths in Sri Lanka during 2017. The disease occurs in epidemic forms, affects both sexes and all age groups, and is observed in urban and rural settings

Keywords: Bone fever; Dandy fever; Dengue haemorragic fever; Dengue shock syndrome.

The disease is caused by dengue virus (DEN), which belongs to genus Flavivirus and family Flaviridae. It is a single stranded, positive sense RNA genome, which is surrounded by an icosahedral nucleo capsid. It contains four serotypes, namely DENV1, DENV-2, DENV-3, and DENV-4. Serotype-1, 2 and 3 and 4, which were first time isolated from Hawaii (1944), New Guinea (1944) and Philippines (1956), respectively. The dengue virus is susceptible to $2 \%$ glutaraldehyde, $70 \%$ ethanol, and $1 \%$ sodium hypochorite. It is sensitive to heat, and low pH can inactivate the virus. Dengue virus remains stable in dried blood and exudates up to several days at room temperature. Antibodies against dengue virus are found in several species of animals, such as monkeys, dogs, horses, goats, buffaloes, pigs, and bats. In urban setting of Thailand, serum samples from domestic dogs were positive for dengue genome by reverse transcriptase-polymerase chain reaction (RT-PCR) technique. Dengue virus was detected in the brain tissues of bats from dengue endemic areas of Mexico and China. The role of animals in transmission cycle of dengue virus to humans needs to be ascertained in order to establish its zoonotic potential. Dengue fever is primarily transmitted by the bite of infected mosquitoes Aedes aegypti. The mosquitoes act as 
the transmitter and the humans serve as the source of infection. The mosquitoes become infected by feeding on the blood of a febrile patient and multiply in the salivary gland. It can transmit the infection to other non-immune person after an extrinsic incubation period of 8-10 days. The eggs of Aedes aegypti can resist desiccation for about 12 months. The mosquito Aedes aegypti bites during day time but more active around sunset and sunrise. The wet long season provides a fertile ground for the breeding of mosquitoes. The arthropod vector of dengue virus flourishes during rainy season. The dengue fever presents three types of transmission cycles, such as sylvatic, rural, and urban.

The disease manifests in four clinical forms, viz.1.high fever, 2. haemorrages (dengue haemorragic fever, 3. circulatory failure (dengue shock syndrome), and 4 . hepatomegaly. The dengue haemorragic fever and dengue shock syndrome are very serious, and carry $40-50 \%$ mortality rate, if not timely treated. The disease has an incubation period of 5 to 9 days, and the patients exhibit a variety of clinical symptoms, such as sudden onset of fever, chills, anorexia, severe headache, vomiting, abdominal pain, malaise, gastrointestinal bleeding, dehydration, profuse sweating, depression, arthralgia, myalgia, photophobia, red eyes, retro-orbital pain, lymphadenopathy, hypotension, bradycardia, sore throat,cough, pneumonia, myocarditis, epistaxis, hematemesis, melena, circumoral cyanosis, fatigue, and erythematous, maculopapular rashes on the body. The high fever remains for 2 to 7 days. Encephalitis may also occur as dengue virus crosses the blood brain barrier. In severe cases, hypovolemia, anoxia, and shock are observed, which results in death within 1 to 2 days. It is mentioned that infants under one year of age are particularly at risk of dying from dengue haemorrhagic fever. The infection is more severe in persons who are immune compromised.

The diagnosis can be confirmed by isolation of virus from the blood during acute stage with fever in tissue cultures. The virus can also be isolated from cerebrospinal fluid (CSF), tissues, and infected mosquitoes. Immunological tests such as compliment fixation, haemagglutination inhibition, and enzyme linked sorbent assay (ELISA) are employed to make the diagnosis. Recently, RT-PCR as a rapid molecular tool to detect and type dengue virus in sera and fresh tissues from patients, is used in many microbiology laboratories. It is advised to create biosafety level 2 facilities while working in the laboratory. The working table must be properly disinfected with $1 \%$ solution of sodium hypochlorite by giving a contact time of 30 minutes. Dengue fever should be differentiated from other vector borne diseases, such as chickungunya fever, eastern equine encephalitis, Japanese encephalitis, malaria, Sindbis fever, Venezuelan equine encephalitis, western equine encephalitis, and West Nile fever by employing standard laboratory techniques. The patient presented at the hospital with the signs of fever, headache, and itchy rash should be investigated for dengue virus infection.

A plethora of measures, such as vector control (spraying of insecticide in artificial reservoir in and around houses, avoid accumulation of water in earthen vessels, plastic bags, broken bottles, flower pots, old tires, trash cans, unused buckets, discarded tins, and proper covering of water containers), prevention of mosquitoes bite by personal protective measures (use of mosquito net, apply repellent cream on exposed body parts, and wearing of full length sleeve shirts), stay indoor for two hours before sunset and after sunrise, wearing of protective clothing by laboratory workers, proper decontamination of all biologic materials before disposal, immunization with tetravalent live attenuated vaccine, and health education to the public (about breeding sites of mosquitoes, mode of transmission, severity of disease and preventive measures) will certainly reduce the incidence of this most important vector borne viral disease.

Presently, no chemotherapeutic agent is available to treat this life threatening disease. However, good supportive therapy, which includes proper bed rest, antipyretics, analgesics, electrolytes, and blood transfusion, is imperative to save the life of patient. Paracetamol can be taken to reduce the body temperature and joint pains. As aspirin and ibuprofen can increase the risk of bleeding, it is advised not to prescribe these drugs to the patients. Acute phase with fever and muscular pain lasts for about 7 to 14 days. However, the full recovery occurs in several weeks. Some physicians have tried the extract of papaya leaf to treat clinical cases of dengue fever. Therefore, the detailed pharmacological and virological studies should be conducted to determine the efficacy of leaves of papaya (Carica papaya) in the management of dengue fever.

Since the present vaccine confers only partial protection against the dengue virus, further research on the development of highly potent, effective, safe and low cost vaccine, which can be easily affordable by poor resource countries, will be rewarding. It is emphasized to elucidate the zoonotic status of dengue virus as antibodies against this arthropod borne virus are already demonstrated in many animals by researchers. 